\title{
Milchsäuregärung der Glucose durch Peptone
}

\section{Doctoral Thesis}

Author(s):

Schlatter, Gottfried

Publication date:

1922

Permanent link:

https://doi.org/10.3929/ethz-a-000091236

Rights / license:

In Copyright - Non-Commercial Use Permitted

Originally published in:

Sammelband ETH-BIB 
Diss. ETH: $295 \mathrm{~B}$

\section{Milchsäuregärung der Glucose durch Peptone}

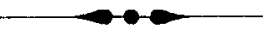

\section{Von der \\ Eidgenössischen Technischen Hochschule in Zürich}

zur Erlangung der

Würde eines Dokfors der technischen Wissenschaften

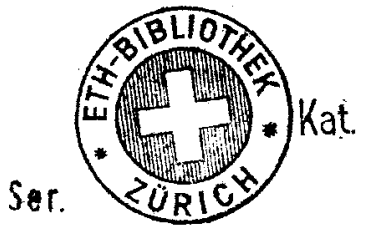

Gottfried Schlatter

dipl. Ingenieur-Chemiker

aus Zurich

No. 295
Referent: Herr Prof. Dr. E. Baur.

Korreferent: Herr Prof. Dr. v. Gonzenbach. 
verbessert wird. Die Gasentwicklung wird entweder ganz verhindert oder verzögert, nie aber vermehrt. Immerhin wäre es nicht ausgeschlossen, daß sich kolloide Stoffe finden lassen, die in geeigneter Konzentration den Effekt zu verstärken vermöchten.

\section{Putride Gärung.}

Die zuckerfreien Kontrollversuche, sowie solche mit Pepton Witte, die sehr lange beobachtet werden müssen, gehen manchmal in Fäulnis über, was sich durch Geruch nach Methylamin verrät. Es war daher geboten, durch eine schärfere Prüfung bei den richtig verlaufenen Milchsäuregärungen sich von der Abwesenheit solcher fremder, von Bakterien ausgehenden Veränderungen zu überzeugen. Pepton Siegfried, wie fast alle Peptone, enthalten Tryptophan, nachzuweisen durch die Rot- und Violettfärbung mit Rohdes ${ }^{1}$ ) Reagens, das aus $20 \mathrm{~g}$ Dimethylaminobenzaldehyd, $500 \mathrm{ccm}$ konz. $\mathrm{HCl}$ und $500 \mathrm{ccm}$ Wasser zusammengesetzt ist. Tryptophan verwandelt sich bei jeder Spur von Fäulnis in Indol, welches durch folgendes Reagens eine Rotfärbung zeigt: $20 \mathrm{~g}$ Dimethylaminobenzaldehyd in $500 \mathrm{ccm}$ Wasser. Die richtig vergorene Lösung, damit versetzt, bleibt farblos, während mit Rohdes Reagens das unveränderte Tryptophan nachweisbar ist. Putride Gärungen bei den richtig verlaufenen Versuchen sind somit ausgeschlossen. Tritt einmal Geruch nach Methylamin auf, so ist auch Indol nachweisbar. Solche Versuche müssen verworfen werden.

\section{Zusammenfassung.}

Die von Emil Baur und E. Herzfeld als ,Gärung ohne Hefe" beschriebene Zersetzung der Glucose unter Bildung einer Säure und eines jodoformgebenden Stoffes ist weiter untersucht worden. Es wird festgestellt, daß Glucose in Lösungen, die Bicarbonat als Puffer enthalten, durch Pepton bei $37^{\circ}$ quantitativ in Milchsäure übergeführt wird. Die Gärung erreicht nach bestimmter Zeit ihr Ende durch einen Flockungsvorgang am Pepton. Das Pepton kann sein eigenes Gewicht an Zucker vergären. Der zeitliche Verlauf der Gärung in Abhängigkeit von der Konzentration der Reaktionsteilnehmer wird festgestellt: Die Gärung kommt um so rascher in Gang, je mehr Pepton anwesend ist. Die Konzentration des Zuckers übt nur cinen geringen Einfluß aus. Eine wichtige Rolle spielt die Gegenwart von Phosphat, indem phosphatfreie Peptone zur Erregung einer Glucose-Milchsäuregärung unvermögend sind. Unbeschadet der Gärkraft kann die Peptonzuckerlösung vor Zusatz des Puffers auf dem Wasserbad erhitzt werden. Während der Gärung und nur während dieser nimmt die Zahl der freien Aminosäuregruppen im Pepton zu. Milchsäurebildende Bakterien sind in der gärenden Lösung nicht nachweisbar.

1) Rohde, Zeitschr. f. physiol. Chem. 44, 161. 1905. 